\title{
Stress Distribution Near Reinforced Circular Hole Loaded by Pin
}

\author{
By Samuel Levy and Frank C. Smith
}

\begin{abstract}
This paper presents a theoretical analysis for the stress distribution in a flat plate near a reinforced circular hole loaded by a pin, and a comparison of the theory with test results for a plate of sandwich construction. It is assumed that an auxiliary reinforcement at the edge of the hole is so rigid that no distortion of the shape of the hole occurs due to the load. The plate is reinforced in the vicinity of the hole by circular "doubler" plates. The theory and experiment are found to be in good agreement.
\end{abstract}

\section{Introduction}

This paper presents a theoretical analysis for the stress distribution in a flat plate near a reinforced circular hole loaded by a pin, as shown in figure 1, and a comparison of the theory with test results for a plate of sandwich construction. It is assumed that an auxiliary reinforcement at the edge of the hole is so rigid that no distortion of the shape of the hole occurs due to the load. The plate is reinforced in the vicinity of the hole by circular "doubler" plates, which increase the thickness by a factor $\beta$. The analysis proceeds from the assumption that the reinforced sheet is in a condition of plane stress. This requires the reinforcement to be symmetrical on both sides of the sheet to eliminate bending stresses, and it requires the thickness to be small compared to the other dimensions so that the stress can be considered constant across the thickness. The stress cannot be assumed constant for points a distance less than one thickness from the boundary between doubler plate and sheet.

Previous work on the problem of a plate with a circular hole loaded by a pin includes a photoelastic investigation by Coker and an empirical analysis by Bickley [1]. ${ }^{2}$ These investigations were confined to unreinforced holes.

\footnotetext{
1 Presented before Seventh International Congress for Applied Mechanies, London, Sept. 1948.

${ }^{2}$ Figures in brackets indicate the literature references at the end of this paper.
}

Closely allied with the problem of the stress distribution in a flat plate near a reinforced circular hole loaded by a pin is that of an unloaded reinforced circular hole in a plate under stress. This problem has been solved approximately by Timoshenko [2], who treated the reinforcement as a curved beam of constant section. Other solutions of the unloaded hole problem based on the plane stress theory were obtained by Sezawa and Kubo [3], by Gurney [4], and by Beskin [5].

Another related problem is that of the stress distribution in a flat plate containing an elliptical region filled with material having a greater stiffness than the material in the rest of the plate. A solution to this problem was obtained by Donnell [6] by using a method that is a generalization of Inglis' solution for the elliptical hole.

In the present paper, use is made of the general plane stress solution in polar coordinates. The solution given is approximate in that it does not give zero stress on the free boundaries of the plate. However, there is reason to believe that for plates with moderately small holes, e. g., plates in which the hole reduces the section of the plate 15 percent or less, this solution gives a good approximation of the stress distribution, particularly in the neighborhood of the hole.

It was decided to check the theory by comparing computed strains with values measured in tests on a specimen of Metalite sandwich construction under concentrated load acting in the plane of the 
plate. It was hoped that the comparison would indicate that reinforcements in this type of construction could be designed rationally on the basis of the plane stress theory.

\section{Boundary Conditions}

The analysis proceeds in two steps. First, a solution is obtained for the stresses and displacements in a circular ring of constant thickness and any inner and outer diameters. Then the solution for the reinforced specimen is obtained by considering the reinforced parts as one such ring and the rest of the sheet as another. Symbols having the subscript " 2 " correspond to the reinforced sheet, whereas symbols having the subscript "1" correspond to the unreinforced sheet. Symbols without a subscript are general and apply to any ring of constant thickness.

The origin of coordinates, figure 1 , is taken at the center of the hole with $x, y$ as rectangular

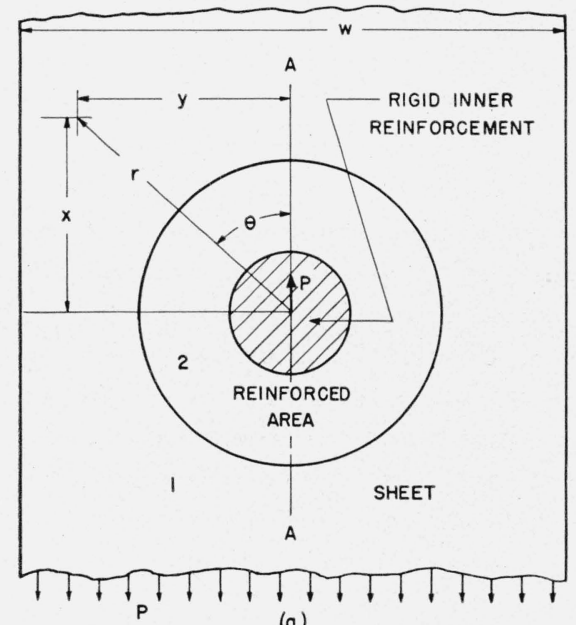

(a)

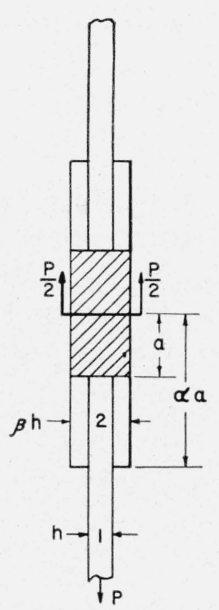

(b)
Figure 1. Problem to be analyzed, (a) plan view of plate, (b) section at $A-A$.

coordinates for points in the sheet and $r, \theta$ as polar coordinates. The positive direction of $x$ will be taken in the direction of the applied load at the hole. The relations between the coordinates are,

$$
\left.\begin{array}{l}
x=r \cos \theta \\
y=r \sin \theta \\
r=\sqrt{x^{2}+y^{2}} \\
\theta=\tan ^{-1} y / x
\end{array}\right\}
$$

Normal and shearing stresses are denoted by the conventional symbols $\sigma$ and $\tau$. Radial displacements are denoted by $u_{r}$ and circumferential displacements by $v_{\theta}$.

Since the edge of the hole is assumed not to deform, the boundary conditions for displacements at the inner edge of the reinforcement can be taken as,

$$
\left.\begin{array}{ll}
u_{\tau 2}=0, & r=a \\
v_{\theta 2}=0, & r=a
\end{array}\right\}
$$

and for forces,

$\left.\begin{array}{c}\beta h \int_{0}^{2 \pi}\left[\sigma_{r 2} \cos \theta-\tau_{r \theta 2} \sin \theta\right]_{(r=a)} a d \theta=-P \\ \beta h \int_{0}^{2 \pi}\left[\sigma_{r 2} \sin \theta+\tau_{r \theta 2} \cos \theta\right]_{(r=a)} a d \theta=0 .\end{array}\right\}$

At the junction between the reinforced and unreinforced sheet, it is necessary that the displacements be the same for both the reinforced and unreinforced sheet, or,

$$
\left.\begin{array}{ll}
u_{r 1}=u_{r 2} ; & r=\alpha a \\
v_{\theta 1}=v_{\theta 2} ; & r=\alpha a .
\end{array}\right\}
$$

It is also necessary that, at the junction between the reinforced and the unreinforced sheet, the normal and shearing forces be the same, or

$$
\left.\begin{array}{ll}
\beta \sigma_{r 2}=\sigma_{r 1} ; & r=\alpha a \\
\beta \tau_{\tau \theta 2}=\tau_{r \theta 1} ; & r=\alpha a
\end{array}\right\} .
$$

The solution of the actual problem, figure 2, a, is the superposition of the solutions for the two subproblems, figure 2, b, and 2, c. For the problem in figure $2, \mathrm{~b}$, the stresses at $r=a$ must satisfy eq 3, and far from the hole, the stresses must remain finite, i. e.;

$$
\sigma_{r 1}, \sigma_{\theta 1} \text {, and } \tau_{i \theta 1} \text { finite } ; r \rightarrow \infty \text {. }
$$

For the problem in figure 2, c, the stresses at $r$ - $a$ must satisfy the condition that the resultant load on the hole is zero

$$
\left.\begin{array}{l}
\int_{0}^{2 \pi}\left[\sigma_{r 2} \cos \theta-\tau_{r \theta 2} \sin \theta\right]_{(\tau=a)} d \theta=0 \\
\int_{0}^{2 \pi}\left[\sigma_{r 2} \sin \theta+\tau_{r \theta 2} \cos \theta\right]_{(r=a)} d \theta=0
\end{array}\right\} ; r=a
$$




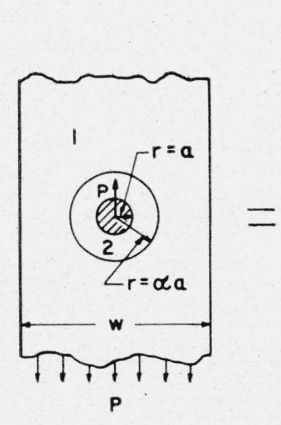

(a)

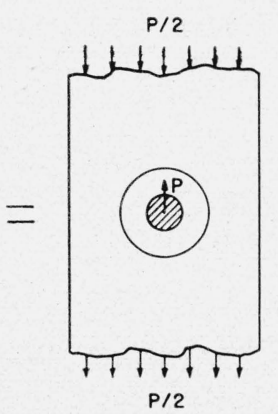

(b)

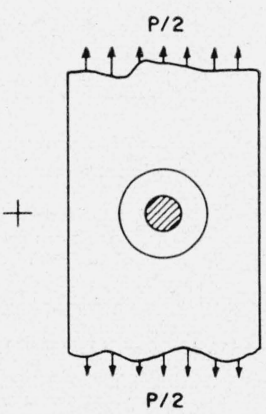

(c)

FIGURE 2. Solution for the actual problem (a) by the superposition of the solutions for the subproblems (b) and $(c)$.

and that, far from the hole, the stress is a uniform tension in the $x$-direction of magnitude $P / 2 w h$, where $w$ is the width of the specimen:

$$
\left.\begin{array}{r}
\sigma_{r 1}=(P / 2 w h) \cos ^{2} \theta \\
\sigma_{\theta 1}=(P / 2 w h) \sin ^{2} \theta \\
\tau_{r \theta 1}=-(P / 4 w h) \sin ^{2} \theta
\end{array}\right\} ; r \rightarrow \infty .
$$

For both the problem in figure $2, \mathrm{~b}$, and the problem in figure $2, \mathrm{c}$, the stresses on the free longitudinal edges of the plate should be zero. Auxiliary stress functions of the type used by Howland [7] might be used to satisfy this boundary condition. This is not done, however, since for moderately small values of the ratio $a / w$ of hole radius to plate width, the stress functions to be used reduce the unbalanced stresses on the free edges to a negligible amount.

\section{Solution for Problem in Figure 2, b}

By using the second line of the general stress function eq 77 [8, p. 114],

$$
\begin{gathered}
\phi=\frac{1-\nu}{4 \pi} M r(\log r) \cos \theta-\frac{M r}{2 \pi} \theta \sin \theta+ \\
\frac{T}{4 \pi r} \cos \theta+\frac{R r^{3}}{4 \pi} \cos \theta
\end{gathered}
$$

where

$$
\nu=\text { Poisson's ratio, }
$$

$M, T, R=$ arbitrary constants to be determined from boundary conditions, it may be shown from eq 34 [8, p. 53] that the equations of equilibrium and of compatibility for

the stresses in a ring of constant thickness are satisfied by

$$
\left.\begin{array}{c}
\sigma_{r}=-\frac{3+\nu}{4 \pi} \frac{M \cos \theta}{r}-\frac{1}{2 \pi} \frac{T \cos \theta}{r^{3}}+\frac{1}{2 \pi} R r \cos \theta \\
\sigma_{\theta}=\frac{1-\nu}{4 \pi} \frac{M \cos \theta}{r}+\frac{1}{2 \pi} \frac{T \cos \theta}{r^{3}}+\frac{3}{2 \pi} R r \cos \theta \\
\tau_{r \theta}=\frac{1-\nu}{4 \pi} \frac{M \sin \theta}{r}-\frac{1}{2 \pi} \frac{T \sin \theta}{r^{3}}+\frac{1}{2 \pi} R r \sin \theta
\end{array}\right\}
$$

By using the relations between strains and stresses given on pages 62 and 63 of [8], it may be shown that the corresponding displacements are

$$
\left.\begin{array}{rl}
u_{r}= & \frac{M}{4 \pi E}\left(\nu^{2}-2 \nu-3\right)(\cos \theta) \log r+\frac{T}{4 \pi E r^{2}}(1+\nu) \cos \theta \\
& +\frac{R r^{2}}{4 \pi E}(1-3 \nu) \cos \theta+\frac{N}{4 \pi E} \cos \theta \\
v_{\theta}= & \frac{M}{4 \pi E}\left(1+2 \nu+\nu^{2}\right) \sin \theta- \\
& \frac{M}{4 \pi E}\left(\nu^{2}-2 \nu-3\right)(\sin \theta) \log r \\
& +\frac{T}{4 \pi E r^{2}}(1+\nu) \sin \theta+\frac{R r^{2}}{4 \pi E}(5+\nu) \sin \theta- \\
& \frac{N}{4 \pi E} \sin \theta
\end{array}\right\}
$$

where

$E=$ Young's modulus,

$N=$ integration constant to be determined from boundary conditions.

Applying the boundary conditions, eq 2, to eq 11 gives

$$
\begin{gathered}
M_{2}\left(\nu^{2}-2 \nu-3\right) \log a+N_{2}+\frac{T_{2}}{a^{2}}(1+\nu)+ \\
R_{2} a^{2}(1-3 \nu)=0
\end{gathered}
$$

and,

$M_{2}\left(1+2 \nu+\nu^{2}\right)+M_{2}\left(3+2 \nu-\nu^{2}\right) \log a-N_{2}+$

$$
\frac{T_{2}}{a^{2}}(1+\nu)+R_{2} a^{2}(5+\nu)=0
$$

Applying eq 3 to eq 10 gives

$$
M_{2}=P / \beta h \text {. }
$$


Applying eq 4 to eq 11 gives

$$
\left.\begin{array}{c}
M_{2}\left(\nu^{2}-2 \nu-3\right) \log \alpha a+N_{2}+\frac{T_{2}}{\alpha^{2} a^{2}}(1+\nu)+ \\
R_{2} \alpha^{2} a^{2}(1-3 \nu)= \\
M_{1}\left(\nu^{2}-2 \nu-3\right) \log \alpha a+N_{1}+\frac{T_{1}}{\alpha^{2} a^{2}}(1+\nu)+ \\
R_{1} \alpha^{2} a^{2}(1-3 \nu),
\end{array}\right\}
$$

and

$$
\left.\begin{array}{c}
M_{2}\left(1+2 \nu+\nu^{2}\right)+M_{2}\left(3+2 \nu-\nu^{2}\right) \log \alpha a-N_{2}+ \\
\frac{T_{2}}{\alpha^{2} a^{2}}(1+\nu)+R_{2} \alpha^{2} a^{2}(5+\nu)= \\
M_{1}\left(1+2 \nu+\nu^{2}\right)+M_{1}\left(3+2 \nu-\nu^{2}\right) \log \alpha a-N_{1}+ \\
\frac{T_{1}}{\alpha^{2} a^{2}}(1+\nu)+R_{1} \alpha^{2} a^{2}(5+\nu) .
\end{array}\right\}
$$

Applying eq 5 to eq 10 gives

$$
\begin{gathered}
\beta\left[-(3+\nu) M_{2}-\frac{2}{\alpha^{2} a^{2}} T_{2}+2 R_{2} \alpha^{2} a^{2}\right]= \\
-(3+\nu) M_{1}-\frac{2}{\alpha^{2} a^{2}} T_{1}+2 R_{1} \alpha^{2} a^{2},
\end{gathered}
$$

and,

$$
\begin{gathered}
\beta\left[(1-\nu) M_{2}-\frac{2}{\alpha^{2} a^{2}} T_{2}+2 R_{2} \alpha^{2} a^{2}\right]= \\
(1-\nu) M_{1}-\frac{2}{\alpha^{2} a^{2}} T_{1}+2 R_{1} \alpha^{2} a^{2} .
\end{gathered}
$$

Applying eq 6 to eq 10 gives

$$
R_{1}=0
$$

Equations 12 to 19 may be solved for the eight constants $\mathrm{M}_{2}, N_{2}, T_{2}, R_{2}, M_{1}, N_{1}, T_{1}$, and $R_{1}$.

\section{Solution for Problem in Figure 2, c}

Reference [4] shows that the equations of equilibrium and compatibility in a ring of constant thickness are satisfied by the stresses

$$
\left.\begin{array}{rl}
\sigma_{r} & =F+K a^{2} / r^{2}+\left(-A-3 C a^{4} / r^{4}-2 D a^{2} / r^{2}\right) \cos 2 \theta \\
\sigma_{\theta} & =F-K a^{2} / r^{2}+\left(A+6 B r^{2} / a^{2}+3 C a^{4} / r^{4}\right) \cos 2 \theta \\
\tau_{r \theta} & =\left(A+3 B r^{2} / a^{2}-3 C a^{4} / r^{4}-D a^{2} / r^{2}\right) \sin 2 \theta,
\end{array}\right\}
$$

where $F, K, A, B, C, D$, are constants to be determined from the boundary conditions. It also shows that the corresponding displacements are

$$
\begin{aligned}
u_{r}= & (r / E)\left\{F(1-\nu)-K(1+\nu) a^{2} / r^{2}+[-A(1+\nu)\right. \\
& \left.\left.-2 \nu B r^{2} / a^{2}+C(1+\nu) a^{4} / r^{4}+2 D a^{2} / r^{2}\right] \cos 2 \theta\right\} \\
v_{\theta}= & (r / E)\left[A(1+\nu)+B(3+\nu) r^{2} / a^{2}+C(1+\nu) a^{4} / r^{4}\right. \\
& \left.-D(1-\nu) a^{2} / r^{2}\right] \sin 2 \theta .
\end{aligned}
$$

Applying the boundary conditions of eq 2 to eq 21 gives

$$
\begin{gathered}
(1-\nu) F_{2}=(1+\nu) K_{2} \\
(1+\nu) A_{2}=-2 \nu B_{2}+(1+\nu) C_{2}+2 D_{2} \\
(1+\nu) A_{2}=-(3+\nu) B_{2}-(1+\nu) C_{2}+(1-\nu) D_{2}
\end{gathered}
$$

Applying eq 4 to eq 21 gives

$$
\begin{aligned}
& (1-\nu) \alpha^{2} F_{2}-(1+\nu) K_{2}=(1-\nu) \alpha^{2} F_{1}-(1+\nu) K_{1}, \\
& -(1+\nu) \alpha^{4} A_{2}-2 \nu \alpha^{6} B_{2}+(1+\nu) C_{2}+2 \alpha^{2} D_{2}= \\
& -(1+\nu) \alpha^{4} A_{1}-2 \nu \alpha^{6} B_{1}+(1+\nu) C_{1}+2 \alpha^{2} D_{1}, \\
& (1+\nu) \alpha^{4} A_{2}+(3+\nu) \alpha^{6} B_{2}+(1+\nu) C_{2}-(1-\nu) \alpha^{2} D_{2}= \\
& (1+\nu) \alpha^{4} A_{1}+(3+\nu) \alpha^{6} B_{1}+(1+\nu) C_{1}-(1-\nu) \alpha^{2} D_{1} .
\end{aligned}
$$

Applying eq 5 to eq 20 gives

$$
\begin{gathered}
\beta \alpha^{2} F_{2}+\beta K_{2}=\alpha^{2} F_{1}+K_{1}, \\
\beta \alpha^{4} A_{2}+3 \beta C_{2}+2 \beta \alpha^{2} D_{2}=\alpha^{4} A_{1}+3 C_{1}+2 \alpha^{2} D_{1}, \\
\beta \alpha^{4} A_{2}+3 \beta \alpha^{6} B_{2}-3 \beta C_{2}-\beta \alpha^{2} D_{2}= \\
\alpha^{4} A_{1}+3 \alpha^{6} B_{1}-3 C_{1}-\alpha^{2} D_{1}
\end{gathered}
$$

Applying eq 8 to eq 20 gives

$$
\begin{gathered}
F_{1}=P / 4 w h, \\
A_{1}=-P / 4 w h, \\
B_{1}=0 .
\end{gathered}
$$

\section{Solution for Problem in Figure 2, a}

Combining eq 10 and 20, we obtain, for the stresses in a ring of constant thickness,

$$
\begin{aligned}
\sigma_{r}= & F+K a^{2} / r^{2}+ \\
& {\left[-(3+\nu) M / 4 \pi r-T / 2 \pi r^{3}-R r / 2 \pi\right] \cos \theta+} \\
& {\left[-A-3 C a^{4} / r^{4}-2 D a^{2} / r^{2}\right] \cos 2 \theta, } \\
\sigma_{r}= & F-K a^{2} / r^{2}+ \\
& {\left[(1-\nu) M / 4 \pi r+T / 2 \pi r^{3}+3 R r / 2 \pi\right] \cos \theta+} \\
& {\left[A+6 B r^{2} / a^{2}+3 C a^{4} / r^{4}\right] \cos 2 \theta, } \\
\tau_{r \theta}= & {\left[(1-\nu) M / 4 \pi r-T / 2 \pi r^{3}+R r / 2 \pi\right] \sin \theta+} \\
& {\left[A+3 B r^{2} / a^{2}-3 C a^{4} / r^{4}-D a^{2} / r^{2}\right] \sin 2 \theta . }
\end{aligned}
$$


The values of the arbitrary constants, $A, B, C$, $D, K, M, R, T$ for the sheet 1 and the reinforcement 2 can be determined as follows: $M_{2}, R_{1}, F_{1}$, $A_{1}, B_{1}$ : From eq $14,19,31,32$, and 33

$$
\left.\begin{array}{l}
M_{2}=P / \beta h \\
R_{1}=0 \\
F_{1}=P / 4 w h \\
A_{1}=-P / 4 w h \\
B_{1}=0
\end{array}\right\}
$$

$M_{1}$ : By combining eq 14, 17 and 18 :

$$
M_{1}=P / h \text {. }
$$

$T_{1}, T_{2}, R_{2}$ : By combining eq $12,13,15,16$, and 17 , and making use of eq 35 and 36 , three simultaneous equations are obtained:

$$
\begin{aligned}
& 2(1+\nu) T_{2}+2(3-\nu) a^{4} R_{2}=-(1+\nu)^{2} a^{2} P / \beta h \\
& 2(1+\nu) T_{2}+2(3-\nu) \alpha^{4} a^{4} R_{2}-2(1+\nu) T_{1}= \\
& \quad(1+\nu)^{2} \alpha^{2} a^{2}(\beta-1) P / \beta h \\
& -2 \beta T_{2}+2 \beta \alpha^{4} a^{4} R_{2}+2 T_{1}=0 .
\end{aligned}
$$

$K_{1}, F_{2}, K_{2}$ : By combining eq 22, 25, and 28 and making use of eq 35

$$
\left.\begin{array}{l}
(1-\nu) F_{2}-(1+\nu) K_{2}=0 \\
(1-\nu) \alpha^{2} F_{2}-(1+\nu) K_{2}+(1+\nu) K_{1}=(1-\nu) \alpha^{2} P / 4 w h \\
\beta \alpha^{2} F_{2}+\beta K_{2}-K_{1}=\alpha^{2} P / 4 w h .
\end{array}\right\}
$$

$C_{1}, D_{1}, A_{2}, B_{2}, C_{2}, D_{2}$ : From eq 23, 24, 26, 27, 29, and 30 , and making use of eq 35 ,

$$
\begin{aligned}
& (1+\nu) A_{2}+2 \nu B_{2}-(1+\nu) C_{2}-2 D_{2}=0 \\
& (1+\nu) A_{2}+(3+\nu) B_{2}+(1+\nu) C_{2}-(1-\nu) D_{2}=0 \\
- & (1+\nu) \alpha^{4} A_{2}-2 \nu \alpha^{6} B_{2}+(1+\nu) C_{2}+2 \alpha^{2} D_{2}- \\
& (1+\nu) C_{1}-2 \alpha^{2} D_{1}=(1+\nu) \alpha^{4} P / 4 w h \\
& (1+\nu) \alpha^{4} A_{2}+(3+\nu) \alpha^{6} B_{2}+(1+\nu) C_{2}- \\
& (1-\nu) \alpha^{2} D_{2}-(1+\nu) C_{1}+(1-\nu) \alpha^{2} D_{1}= \\
- & (1+\nu) \alpha^{4} P / 4 w h \\
\beta & \alpha^{4} A_{2}+3 \beta C_{2}+2 \beta \alpha^{2} D_{2}-3 C_{1}-2 \alpha^{2} D_{1}=-\alpha^{4} P / 4 w h \\
\beta & \alpha^{4} A_{2}+3 \beta \alpha^{6} B_{2}-3 \beta C_{2}-\beta \alpha^{2} D_{2}+3 C_{1}+ \\
& \alpha^{2} D_{1}=-\alpha^{4} P / 4 w h
\end{aligned}
$$

Combining eq 11 and 21, we obtain for the displacements in a ring of constant thickness,

$$
\begin{aligned}
u_{r}= & (r / E)\left[F(1-\nu)-K(1+\nu) a^{2} / r^{2}\right]+ \\
& {[(\cos \theta) / 4 \pi E]\left[M\left(\nu^{2}-2 \nu-3\right) \log r+\right.} \\
& \left.T(1+\nu) / r^{2}+R r^{2}(1-3 \nu)+N\right]+ \\
& {[(r \cos 2 \theta) / E]\left[-A(1+\nu)-2 \nu B r^{2} / a^{2}+\right.} \\
& \left.C(1+\nu) a^{4} / r^{4}+2 D a^{2} / r^{2}\right] \\
v_{\theta}= & {[(\sin \theta) / 4 \pi E]\left[M(1+\nu)^{2}+M\left(3+2 \nu-\nu^{2}\right) \log r+\right.} \\
& \left.T(1+\nu) / r^{2}+R r^{2}(5+\nu)-N\right]+ \\
& {[(r \sin 2 \theta) / E]\left[A(1+\nu)+B(3+\nu) r^{2} / a^{2}+\right.} \\
& \left.C(1+\nu) a^{4} / r^{4}-D(1-\nu) a^{2} / r^{2}\right]
\end{aligned}
$$

The additional arbitrary constants, $N_{1}, N_{2}$, are obtained by combining eq $12,13,15$, and 16 with eq 35 giving:

$$
\left.\begin{array}{rl}
N_{1}= & -2(1+\nu)\left(\alpha^{2}-1\right) a^{2} R_{2}+[(1+\nu) \beta+ \\
& 2 \beta(3-\nu) \log \alpha a-2(3-\nu) \log \alpha] \\
& (1+\nu) P / 2 \beta h \\
N_{2}= & 2(1+\nu) a^{2} R_{2}+[1+\nu+2(3-\nu) \log a] \\
& (1+\nu) P / 2 \beta h
\end{array}\right\}
$$

\section{Comparison of Analysis With Experiment}

The specimen, figures 3 and 4, was furnished for these tests by Chance-Vought Aircraft, Dallas, Texas. It was of Metalite construction, consisting of two sheets of $0.013-i n$. 24S-T81 Alclad aluminum alloy bonded to a 0.5 -in core of balsa wood, which had been cut with the grain normal to the sheet. The edges of the specimen were reinforced by pairs of $3 / 8-$ by $3 / 4^{-}$by $1 / 8$-in. $24 \mathrm{~S}-\mathrm{T}$ aluminum alloy angles bonded to the sheet.

The concentrated load was applied by a steel pin, of 1.25-in. diameter, making a snug, but turning, fit in a hole reamed in a steel bushing. The Metalite was reinforced to a radius of $5 \mathrm{in}$. from the center of the loading pin. A detail of the reinforcement is shown at section $\mathbf{A}-\mathbf{A}$, figure 3. The reinforcement to a radius of $2.5 \mathrm{in}$. was considered heavy enough to prevent distortion inside this circle. 


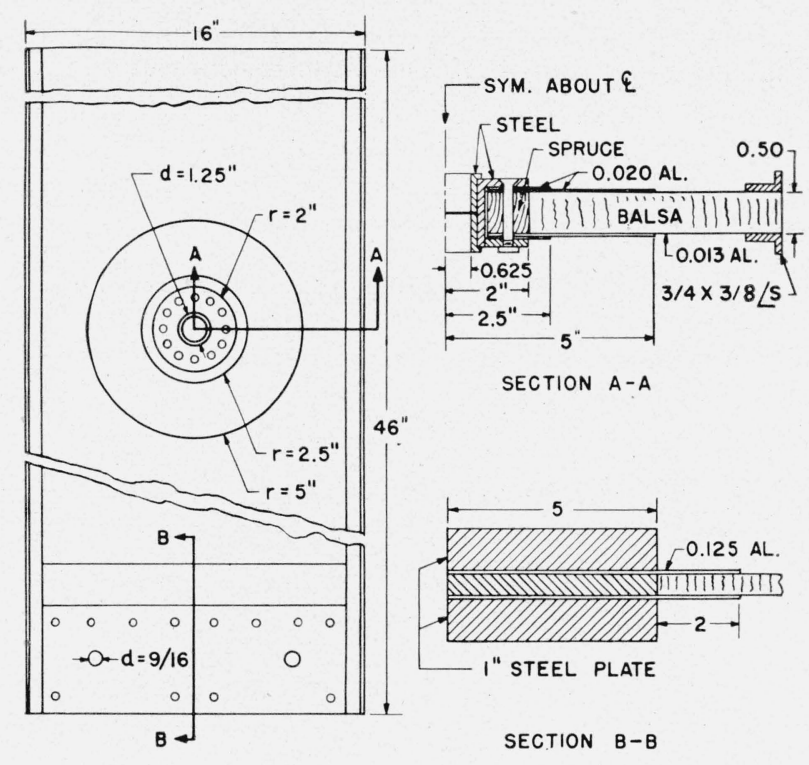

FiguRE 3. Specimen tested, showing reinforcement near hole

The following constants were taken from the specimen and were used in the analysis of the experiment:

$$
\begin{aligned}
a & =2.5 \mathrm{in} . \\
\alpha a & =5 \mathrm{in} . \\
h & =0.026 \mathrm{in} . \\
\beta h & =0.066 \mathrm{in} . \\
\nu & =0.3 .
\end{aligned}
$$

The effective width, $w$, of the panel was obtained by dividing the gross cross section of the panel, including edge angles, by the sheet thickness, with the result

$$
w=31.56 \text { in. }
$$

The arbitrary constants were determined by solving the simultaneous equations in section $\mathrm{V}$, with the result:

$$
\begin{array}{ll}
A_{1}=-0.3049 P & A_{2}=-0.1295 P \\
B_{1}=0 & B_{2}=-0.001977 P \\
C_{1}=1.194 P & C_{2}=0.06584 P \\
D_{1}=-0.7572 P & D_{2}=-0.1275 P \\
F_{1}=0.3049 P & F_{2}=0.1425 P \\
K_{1}=0.426 P & K_{2}=0.0768 P \\
M_{1}=38.46 P & M_{2}=15.15 P
\end{array}
$$

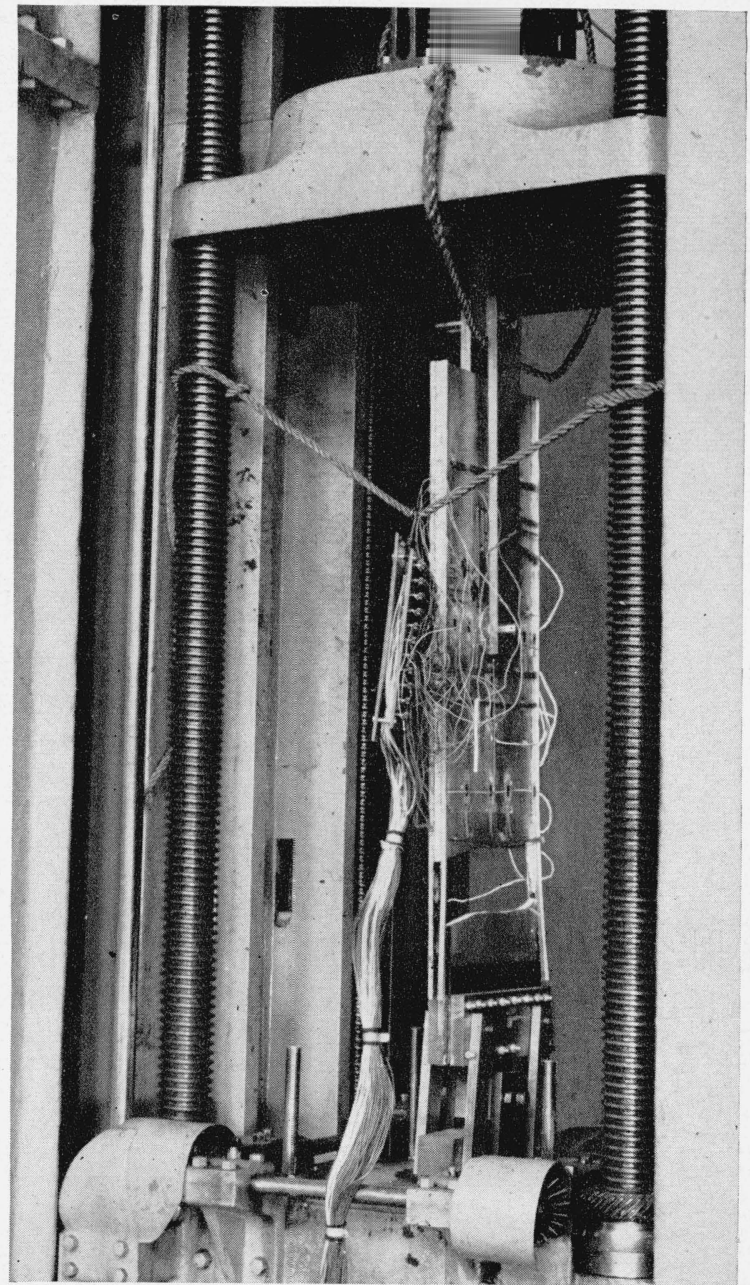

FIGURE 4. Specimen in testing machine after failure.

$$
\begin{array}{ll}
N_{1}=208.0 P & N_{2}=63.01 P \\
R_{1}=0 & R_{2}=0.09215 P \\
T_{1}=-321.7 P & T_{2}=-69.04 P
\end{array}
$$

The stress in the unreinforced region $1(r>5)$ was computed by substituting the constants with subscript 1 into eq 34 ; whereas the stress in the reinforced region $2(2.5<r<5)$ was similarly computed using constants with subscript 2 .

The radial and circumferential strains were computed from the stresses by the well-known formulas

$$
\left.\begin{array}{l}
\epsilon_{r}=\frac{1}{E}\left(\sigma_{r}-\nu \sigma_{\theta}\right) \\
\epsilon_{\theta}=\frac{1}{E}\left(\sigma_{\theta}-\nu \sigma_{r}\right)
\end{array}\right\}
$$


taking

$$
\begin{aligned}
E & =10.6 \times 10^{6} 1 \mathrm{~b} / \text { in. }^{2} \\
\nu & =0.3
\end{aligned}
$$

Figure 5 shows the measured strains at a load of $10,000 \mathrm{lb}$, and figure 6 shows a comparison between measured and computed longitudinal strains for the longitudinal and transverse centerlines and for a transverse section $12 \mathrm{in}$. below the center of the hole. The computed maximum strain, just outside the reinforcement, differed only about 2 percent from the measured strain. Larger differences were found elsewhere. The agreement is as good as could be expected in view of the assump-

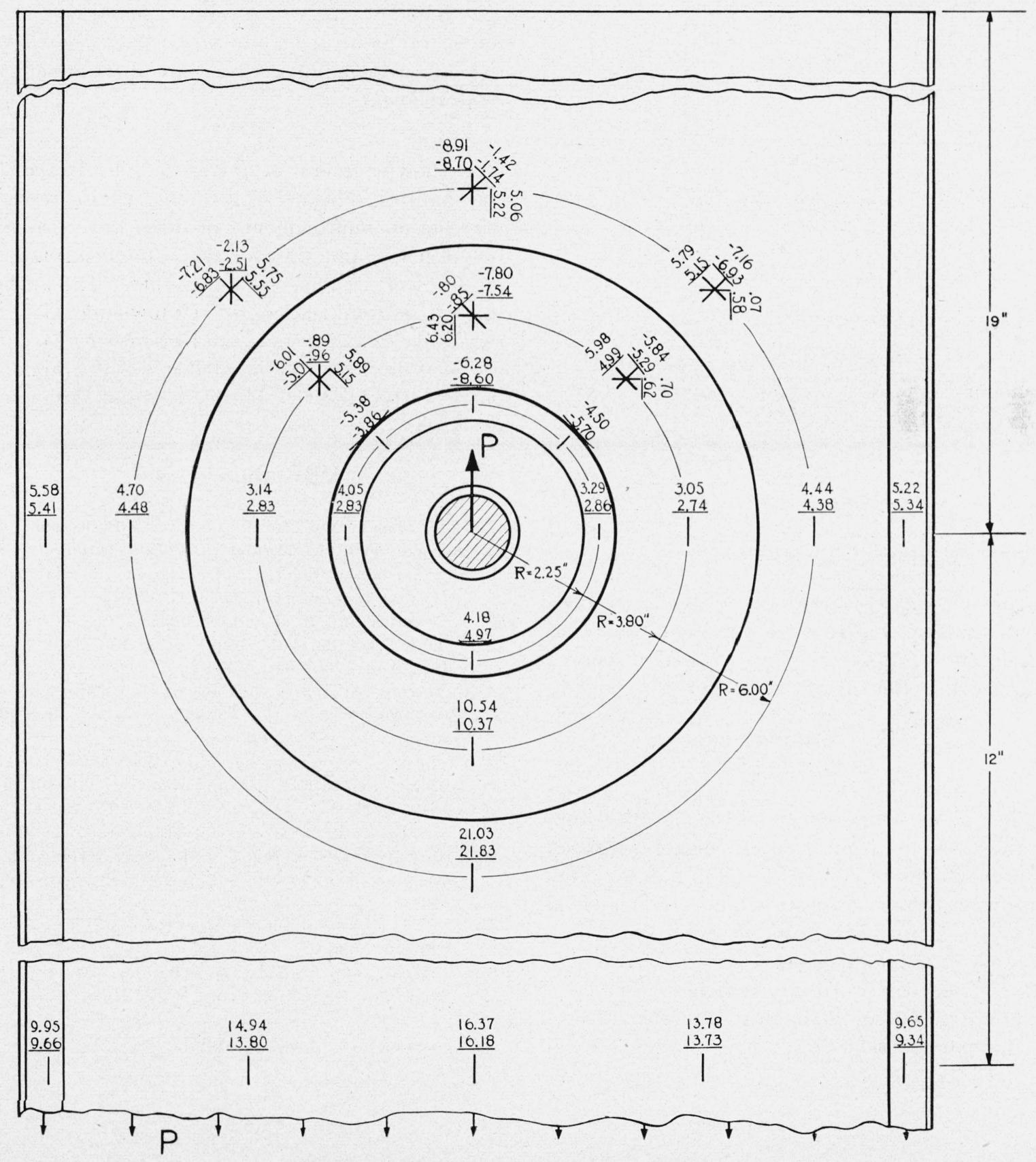

Figure 5. Strain distribution at load of 10,000 lb (strains $\left.\times 10^{4}\right)$.

Underlined strain values are based on measurements on front of specimen; other strain measured on back of specimen. 

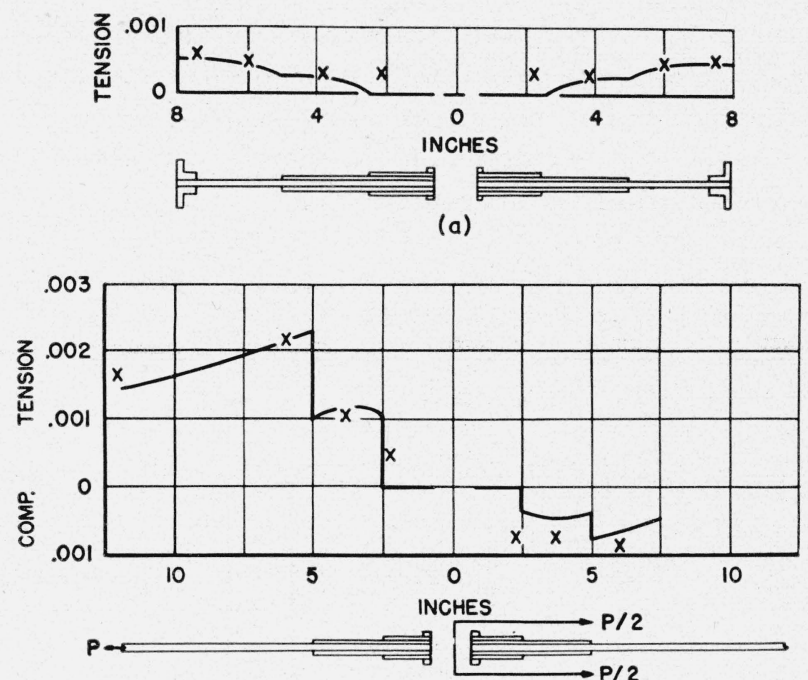

(b)

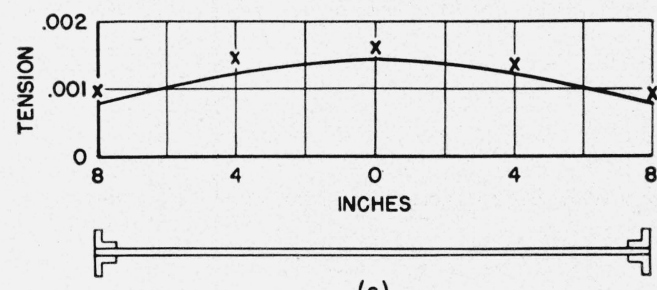

(c)

Figure 6. Average longitudinal strain at 10,000-lb load.

$\mathrm{X}$, Measured; calculated. (a) Transverse center line; (b) longitudinal center line; (c) transverse line 12 in. below center of hole.

tions of the theory that a state of plane stress existed, that the central area was rigid, and that the hole and reinforcement were small compared to the width of the specimen.

\section{Conclusions}

It is concluded that:

1. The plane stress theory may be used to compute the stress distribution in the neighborhood of a reinforced circular hole loaded by a pin in a plate of sandwich construction, in which practically all the load is carried by sheets of metal bonded to each other or to a light-weight core and in which a relatively rigid central reinforcement is used.

2. For large values of the ratio of plate width to hole diameter and with a nearly rigid central reinforcement for applying the load, the solution can be expected to be accurate in all parts of the plate.

3. For moderate values of plate width, the results for the stresses are not of very satisfactory accuracy near the free edges of the plate, but are reliable in the neighborhood of the hole near the places of largest stress.

4. The presence of the reinforcement is likely to move the point of highest stress from the edge of the hole to a point in the plate just outside the reinforcement.

Acknowledgment is made to the Bureau of Aeronautics, Navy Department, whose research projects on reinforcement of holes have provided the impetus and the necessary financial support for the work reported in this paper. The authors take this opportunity to acknowledge also the assistance of members of the Engineering Mechanics Section of the National Bureau of Standards, particularly Albert E. McPherson and Wilhelmina D. Kroll.

\section{References}

[1] E. G. Coker and L. N. G. Filon, Photo-elasticity, p. 524 to 530 (Cambridge Univ. Press, London, 1931).

[2] S. Timoshenko, Strength of materials, part II, p. 457 (D. Van Nostrand Co., Inc., New York, N. Y., 1930).

[3] K. Sezawa and K. Kubo, Stresses in a plate with a flanged circular hole, Rept. Aero. Res. Inst. Tokyo Imp. Univ., No. 84 (1932).

[4] C. Gurney, An analysis of the stresses in a flat plate with reinforced circular hole under edge forces, Brit. Res. Memo. No. 1834 (1938).

[5] L. Beskin, Strengthening of circular holes in plates under edge loads, J. Appl. Mech., p. A-140 (Sept. 1944).

[6] L. H. Donnell, Stress concentrations due to elliptical discontinuities in plates under edge forces (Th. v. Karman Anniversary Volume, Calif. Inst. Tech., 1941).

[7] R. C. J. Howland, Phil. Trans. Roy. Soc. London [A] 229, 49 (1930).

[8] S. Timoshenko, Theory of elasticity (McGraw-Hill Book Co., Inc., New York, N. Y., 1934).

Washington, June 3, 1948. 